\title{
Zwischen König, Staat und Nation. Der Fall der »Nationalen Gesellschaft«, 1894-1897
}

\author{
von \\ VAIOS KALOGRIAS
}

\begin{abstract}
Nach dem erfolgreichen Unabhängigkeitskampf gegen das Osmanische Reich (1821-1829) spielte das griechische Militär eine zentrale Rolle im Leben des neuen und "souveränen "Staatswesens. Bis zum Ende des neunzehnten Jabrhunderts verstand es sich als das unentbehrliche Instrument zur $\gg$ Befreiung der unterjochten Griechen "im Osmanischen Reich und zur Verwirklichung der "Großen Idee" (Megali Idea), also der Zusammenfassung aller Griechen auf dem Balkan und in Kleinasien zu einem neobyzantinischen griechischen Reich. Zwar war das Militär dem Königshaus und dem Staat untergeordnet. Doch im Laufe der Zeit entstanden konspirative Zirkel im Offizierskorps, die politische Forderungen stellten. Im Fall der 1894

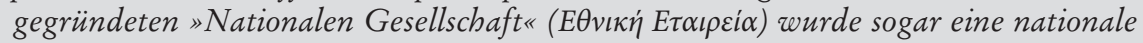
Krise heraufbeschworen: Die von Subalternoffizieren ins Leben gerufene Organisation forderte die Autorität der Krone und des Staates heraus, indem sie das »Recht" beanspruchte, die »wabren Interessen "der Nation zu vertreten. Die "Nationale Gesellschaft "bildete einen Staat im Staate und übte großen Einfluss aus. Mit ibrem Engagement für eine militärische Lösung der Kreta-Frage im Jabre 1897 trug sie zur Eskalation der Beziehungen zwischen Athen und Konstantinopel und zum Ausbruch des griechisch-türkischen Kriegs bei.
\end{abstract}

Im letzten Viertel des neunzehnten Jahrhunderts stand Griechenland vor großen außenpolitischen und wirtschaftlichen Herausforderungen: Die Verschärfung der nationalstaatlichen Antagonismen in den europäischen Teilen des Osmanischen Reichs - etwa in Makedonien und auf Kreta - und die anschwellende Wirtschaftskrise, die 1893 zum Staatsbankrott führte, waren die Hauptmerkmale dieser Zeit. Innenpolitisch blieb die Institution der Monarchie, mit dem gemäßigten und stets auf Vernunft bedachten Georg I. (1845-1913) an der Spitze, unangetastet. Die liberale Reformpolitik des angesehenen und westorientierten Ministerpräsidenten Charilaos Trikoupis (1832-1896) leitete sogar die - bescheidene - Industrialisierung des überwiegend agrarisch geprägten Landes ein. Seine Reformen jedoch - etwa in Verwaltung und Heer - riefen starke Reaktionen innerhalb der griechischen Gesellschaft hervor, da sie die Privilegien der alten Eliten beschnitten und den sozialen Aufstieg des Handelsund Wirtschaftsbürgertums ermöglichten. Die auf eine Modernisierung abzielenden Reformen hatten zur Folge, dass sich die Lage der ärmeren Schichten zusehends verschlechterte, so dass der charismatische Trikoupis auf die Unterstützung der »kleinen Leute - Bauern und städtische Arbeiter - kaum zählen konnte. Sein politischer Gegenspieler, Theodoros Deligiannis (1826-1905), ein 
geschickter Demagoge, profilierte sich indessen als Beschützer von »Nation, Vaterland und Religion «. Seine »Nationale Partei« wurde zur politischen Heimat aller Unzufriedenen, einschließlich der alten Eliten. Während Trikoupis vom aufstrebenden Bürgertum und der geistigen, reformorientierten Elite unterstützt wurde, setzte Deligiannis auf die bewährten Mittel von Klientelismus und Irredentismus ${ }^{1}$.

Eine einflussreiche Gesellschaftsgruppe, die in den Strudel der Auseinandersetzung zwischen Trikoupis und Deligiannis geriet, war das Offizierskorps. Seit der Gründung des griechischen Königreichs 1831 spielte die Armee eine wichtige Rolle für die »Regelung « innenpolitischer Angelegenheiten. Im September 1843 beispielsweise zwang sie König Otto von Wittelsbach (1815-1867) dazu, eine Verfassung zu verabschieden. Ihre Intervention wies in diesem konkreten Fall »progressive « Züge auf, da sie die absolutistische Herrschaftsform des bayerischen Monarchen beendete ${ }^{2}$. Darüber hinaus verstanden sich die Mitglieder des Offizierskorps - in Übersteigerung ihrer traditionellen Rolle

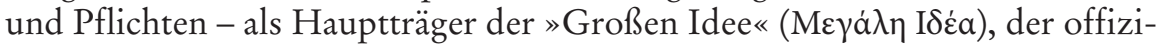
ellen nationalstaatlichen Ideologie. Diese sah die Vereinigung aller im Osmanischen Reich lebenden Griechen mit dem griechischen Königreich und die Schaffung eines hellenischen Großstaates vor. Um dies zu erreichen, übten die Offiziere Druck auf die Athener Regierungen aus, Maßnahmen zur Reorganisierung von Armee und Flotte zu treffen. Die »Befreiung der versklavten Brüder «-vor allem in Makedonien und auf Kreta - hatte für sie absolute Priorität ${ }^{3}$.

Allerdings sollte die Erfüllung des griechischen Irredentismus im Rahmen der bestehenden gesellschaftlichen Ordnung erfolgen. Loyalität gegenüber dem Königshaus sowie die Kooperation mit den politischen Parteien und den übrigen staatstragenden Institutionen waren für die Offiziere notwendig zur Verwirklichung der »Großen Idee«. In der Tat bestand in diesem Punkt weitgehende Übereinstimmung zwischen den verschiedenen gesellschaftlichen Gruppen. Das Königshaus, die Parteien, die Orthodoxe Kirche, das Wirtschaftsbürgertum, die Intellektuellen - darunter prominente Historiker vom Schlage eines Konstantinos Paparigopoulos ${ }^{4}$ - und sogar Frühsozialisten waren von der Idee eines Großreichs - mit Konstantinopel als Hauptstadt - beseelt. Dieses nationale »Projekt« vereinte unterschiedliche politische Strömun-

Zum Reformwerk Trikoupis' und zu seiner politischen Auseinandersetzung mit Deligiannis

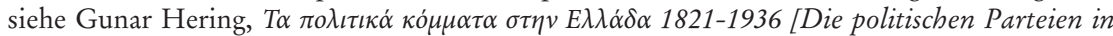
Griechenland 1821-1936], Bd. 1, Athen 2004, S. 558-561, 567-569. Richard Clogg, Geschichte Griechenlands im 19. und 20. Jahrbundert. Ein Abriß, Köln 1997, S. 89-90. Apostolos Vakalopulos, Griechische Geschichte von 1204 bis heute, Köln 1985, S. 165-172.

2 Pavlos Tzermias, Nengriechische Geschichte. Eine Einführung, 3. überarb. und erw. Aufl., Tübingen 1999, S. 96.

3 Zur Rolle der Armee in der Innenpolitik siehe Thanos Veremis, The Military in Greek Politics: From Independence to Democracy, London 1997.

4 Konstantinos Paparigopoulos (1815-1891) war Begründer der neugriechischen national orientierten Historiographie. In seinem fulminanten Werk »Geschichte der Hellenischen Nation« betonte er die Kontinuität des »Griechentums « von der Antike bis in die Gegenwart. Allerdings verstand er darunter eine »kulturelle Kontinuität«, vgl. Tzermias, Nengriechische Geschichte (wie Anm. 2), S. 14-15. 
gen und gesellschaftliche Schichten. Es legitimierte im Grunde die Existenz des von »Gnaden« der Großmächte errichteten Nationalstaats und rechtfertigte alle sozialen Nöte der Gegenwart mit dem Versprechen einer zukünftigen Utopie $^{5}$.

Die innere Geschlossenheit jedoch begann Anfang der 1890er Jahre zu zerbröckeln. Griechenland, inmitten einer schweren Wirtschaftskrise, stand weit davon entfernt, die »Große Idee« auf Kosten des - wohlgemerkt mächtigeren - Osmanischen Reichs zu verwirklichen. Unzufriedenheit und Frustration über die "passive " Haltung des Königshauses und der politischen Parteien gegenüber wichtigen Ereignissen - etwa der »Anschluss « der autonomen Provinz Ost-Rumelien an Bulgarien im Jahr 1885 - breiteten sich aus. In national gesinnten Kreisen des Offizierskorps setzte sich allmählich die Überzeugung durch, dass »etwas getan werden müsse«: Da der Staat offensichtlich weder willens noch in der Lage sei, seine nationale Aufgabe wahrzunehmen, habe die Nation mittels nichtstaatlich kontrollierter patriotischer Privatvereinigungen und -akteure für die Befreiung der »unerlösten Brüder « zu sorgen. Dies bedeutete die Gründung einer von Staat und Regierung unabhängigen Organisation als Vehikel zur Umsetzung der nationalen Forderungen. Die Initiative dazu ging von einigen Subalternoffizieren (Leutnants und Oberleutnants), unter anderem L. Fotiadis, G. Souliotis, Konstantinos Pallis und Pavlos Melas, aus ${ }^{6}$. Tatsächlich gelang es ihnen, innerhalb kürzester Zeit die Fundamente einer Geheimorganisation zu legen, die großes Aufsehen erregte. Im November 1894 wurde die Organisation unter dem Namen »Nationale Gesellschaft «

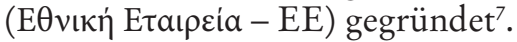

In diesem Beitrag werden Ziele, Strukturen und Aktivitäten der EE beschrieben. Vor allem wird ihr Loyalitätsverhältnis gegenüber den staatstragenden Institutionen (König, Parteien, Armee) untersucht und dabei herausgearbeitet, wie sie von einer kleinen Geheimorganisation zu einem öffentlichen machtpolitischen Faktor ersten Ranges aufstieg.

Über die »Nationale Gesellschaft« ist die Quellenlage sehr dürftig8. Weder das erhalten gebliebene Schrifttum der Organisation noch die Erinnerungen einzelner Mitglieder geben Aufschluss über ihre geheimen Aktivitäten. Gesichert ist: Als Katalysator für die Bildung dieser Gruppe wirkte die Untergrundtätigkeit der 1893 ins Leben gerufenen »Inneren Makedonischen Revolutionären Organisation « (IMRO) im osmanischen Makedonien?. Das Ziel der bulgarisch geprägten IMRO, einen vom Sultan unabhängigen makedonischen Staat - mit Thessaloniki als Hauptstadt - zu errichten, alarmierte die Anhänger

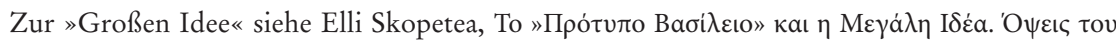

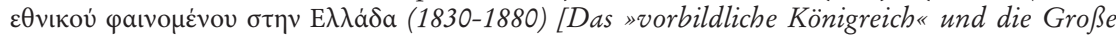
Idee. Aspekte des nationalen Problems in Griechenland (1830-1880)], Athen 1988.

6 Vakalopulos, Griechische Geschichte (wie Anm. 1), S. 172.

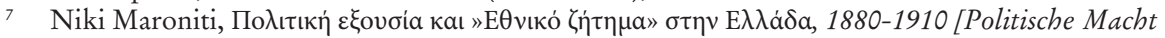
und "Nationale Frage in Griechenland, 1880-1910], Athen 2009, S. 105.

8 Das Standardwerk zu diesem Thema ist immer noch die Studie von Georgios Lyritzis, H E $\theta v ı$ ń

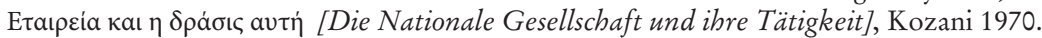

9 Zur Gründung und Tätigkeit der IMRO siehe Duncan Perry, The Politics of Terror. The Macedonian Liberation Movements, 1893-1903, Durham 1988. 
der »Großen Idee « in Athen. Diese sahen in der IMRO eine ernstzunehmende Gefahr für die eigenen Nationalansprüche. Deshalb forderten griechische Offiziere die Entsendung bewaffneter Einheiten nach Makedonien. Durch diese begrenzte militärische Intervention, so glaubten sie, würden Armee und $\mathrm{Na}$ tion aufgerüttelt sowie der Staat gezwungen werden, zu seiner »nationalen Pflicht« zu stehen und militärische Vorbereitungen zu treffen ${ }^{10}$. In der Tat gelang es der »Gesellschaft «, einen Teil ihrer Forderungen durchzusetzen - mit verheerenden Folgen für Griechenland, doch davon später mehr. Mit anderen Worten: Die »Nationale Gesellschaft « war das Gegenstück zur IMRO. Sie war quasi die griechische Antwort auf den großbulgarischen Nationalismus, auf die so genannte »slawische Gefahr«, welche das »Griechentum « in Makedonien bedrohte ${ }^{11}$. Doch die Ambitionen der »Nationalen Gesellschaft« beschränkten sich nicht ausschließlich auf Makedonien.

Zwar hatte es in der Vergangenheit illegale und konspirative Militärzirkel gegeben. Doch keine Geheimorganisation hatte die legitimen Repräsentanten von Staat und Nation auf die Art herausgefordert, wie es die »Nationale Gesellschaft « tat - obwohl sie keine revolutionären Umwälzungen nach dem Beispiel von 1843 forderte ${ }^{12}$. Zur Spitze der »Gesellschaft« gehörten jüngere Offiziere wie Leutnant Pavlos Melas (1870-1904), Panagiotis Daglis (18531924) und Ioannis Metaxas (1871-1941). Alle drei bestimmten die Geschichte Griechenlands in der ersten Hälfte des zwanzigsten Jahrhunderts: Der in den Athener großbürgerlichen Kreisen hochangesehene Melas starb als Freischärler und Märtyrer im »Makedonischen Kampf«(1904-1908). Sein Vater Michail Melas (1833-1897), ein respektables Mitglied der Athener Gesellschaft, wurde im Januar 1897 Vorsitzender des »legalen « Zweigs der Organisation ${ }^{13}$. Daglis wurde Mitglied der antiroyalistischen Gegenregierung von Thessaloniki im Herbst $1916^{14}$. Metaxas errichtete am 4. August 1936 eine Diktatur nach nationalsozialistischem und faschistischem Vorbild und führte Griechenland im Krieg gegen die Achsenmächte ${ }^{15}$.

In der Anfangsphase zählte die »Nationale Gesellschaft« etwa sechzig Mitglieder, ausschließlich Subalternoffiziere. Das Offizierskorps bildete die exklusive Rekrutierungsbasis der Organisation, einfache Soldaten durften nicht angeworben werden. Allerdings billigten nicht alle Offiziere die Initiative der

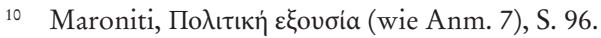

11 Der makedonische Hintergrund der "Nationalen Gesellschaft « wird in den Schriftzeugnissen

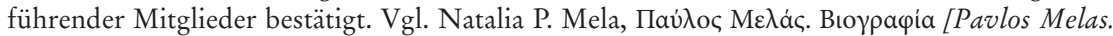
Biografie], 2. Aufl., Athen-Giannena 1992, S. 60.

12 Im September 1843 zwang eine Militärrevolte König Otto zur Verabschiedung einer Verfassung. Somit ging die absolutistische Ära der so genannten »Bayernherrschaft« zu Ende. Siehe I.A.

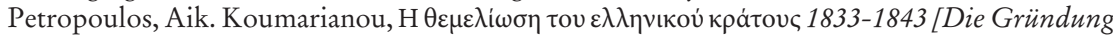
des griechischen Staates 1833-1843], Athen 1982, S. 249-255.

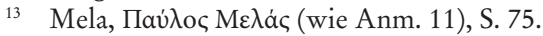

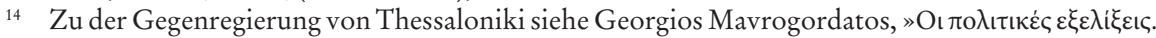

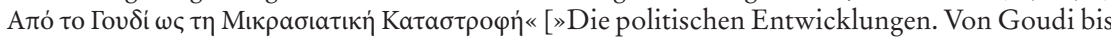

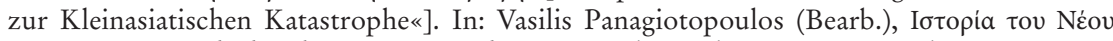

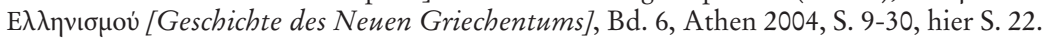

15 Zur Biografie Metaxas' siehe Joachim G. Joachim, Ioannis Metaxas. The Formative Years 18711922, Mannheim-Möhnesee 2000. 
Gründungsmitglieder, und die Organisation repräsentierte keineswegs den »Willen« oder den »Geist « des gesamten Offizierskorps. Hier spielte eine große Rolle, dass es den Angehörigen des Offizierskorps von ihrem rechtlichen Status her nicht gestattet war, Mitglied eines Geheimbundes zu sein. Von einer Spaltung des Offizierskorps in Mitglieder beziehungsweise Anhänger und Gegner der »Gesellschaft « zu sprechen, wäre jedoch übertrieben. Eine »Gegenbewegung « existierte nicht. Gerade viele ranghohe Offiziere sympathisierten mit der Geheimgesellschaft. Militärische Disziplinarmaßnahmen gegen Mitglieder wurden trotz der anderweitigen Rechtslage nicht angewandt, wobei auch die Angst vor Unruhen innerhalb der Armee, die solchen Bestrafungen folgen könnten, sicherlich von hoher Bedeutung war ${ }^{16}$. Und schließlich bestand eine jahrelange Tradition konspirativer Tätigkeit im Offizierskorps ${ }^{17}$, auf welche die Mitglieder der »Gesellschaft « zurückblicken und dadurch ihre Aktivitäten »legitimieren « konnten.

Bis Herbst 1896 hatte die »Gesellschaft « 56 Ortsgruppen in Griechenland und $83 \mathrm{im}$ Ausland (etwa in Alexandria in Ägypten) gegründet ${ }^{18}$. Nach ihrem - allerdings modifizierten - Gründungsstatut trat sie »Im Namen Gottes und des Vaterlands « für die Stärkung des Nationalbewusstseins im »freien Griechenland « und die Förderung des Freiheitsgedankens bei den »unterjochten Brüdern « im Osmanischen Reich ein. Die Aufnahme neuer Mitglieder setzte absoluten Gehorsam, eiserne Disziplin und strikte Geheimhaltung voraus, so dass eine Aufdeckung der Organisation durch staatliche Stellen verhindert wurde. Nach Artikel 5 des Statuts der »Nationalen Gesellschaft« war es den Angehörigen des Königshauses nicht gestattet, der Organisation beizutreten. Regierungsmitglieder hingegen durften der Organisation angehören, so lange sie keinen Ministerposten bekleideten. Aus konspirativen Gründen durften die einfachen Mitglieder die Namen der führenden Köpfe nicht erfahren, welche das Leitungskomitee, die so genannte »unsichtbare Kraft «, darstellten. Es gab zwei Kategorien von Mitgliedern: eine höhere (»Genossen«) und eine niedrigere (»Brüder«). Mitgliedern war es nicht gestattet, die Mitgliedschaft zu kündigen, auf »Verrat « an der Organisation stand der $\operatorname{Tod}^{19}$. Als Vorbild für die strengen Einweihungsrituale der »Nationalen Gesellschaft « dienten jene der

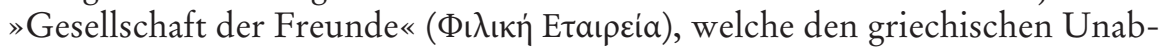
hängigkeitskampf (1821-1829) vorbereitet hatte ${ }^{20}$. So wurde eine Brücke zu nationalrevolutionären Freiheitstraditionen geschlagen und damit historische Kontinuität demonstriert.

War die »Nationale Gesellschaft« anfangs eine geheime Militärorganisation, die ausschließlich Offiziere rekrutierte, so strebte sie bald an, ihre Ziele und

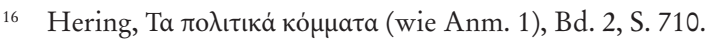

17 Beispielsweise die Beteiligung aktiver Offiziere an der Revolte vom September 1843.

18 Die Mitglieder im Ausland wurden innerhalb der dort ansässigen griechischen Gemeinden

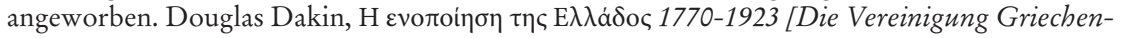
lands 1770-1923], 2. Aufl., Athen 1984, S. 229.

19 Ioannis Zelepos, Die Ethnisierung griechischer Identität 1870-1912. Staat und private Akteure vor dem Hintergrund der »Megali Idea", München 2002, S. 194.

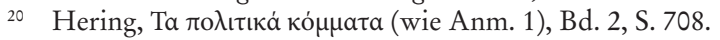


Ideen gesellschaftlich zu verankern. Dadurch erwartete sie die Anerkennung ihrer nationalen Führungsrolle ${ }^{21}$. Dies bedeutete die Öffnung der Organisation für zivile Mitglieder. Wann genau der entsprechende Beschluss gefasst wurde, ist nicht mehr eindeutig nachweisbar. Im Frühjahr 1896 begannen die Verantwortlichen jedenfalls mit der Anwerbung »angesehener Persönlichkeiten«. Als wichtige Verbündete der zum Handeln entschlossenen Offiziere kamen führende Journalisten, Intellektuelle, Künstler, Literaten, Universitätsprofessoren, höhere Beamte und Politiker in Frage - mit einem Wort das Bildungsbürgertum. Sie alle waren dank ihrer Presseagitation und ihrer Prominenz in der Lage, die Bevölkerung für die Ziele der Organisation zu gewinnen und zu mobilisieren. Zusammen mit den ebenfalls dem Bildungsbürgertum zugerechneten Offizieren stellten sie ein besonderes Milieu dar, das sich das Recht nahm, Griechenlands »wahre Interessen« allein zu vertreten.

Unter den zivilen Mitgliedern befanden sich die Schriftsteller und Dichter Grigorios Xenopoulos (1867-1951), Andreas Karkavitsas (1865-1922), Kostis Palamas (1859-1943) und Pavlos Nirvanas (1866-1937), die Universitätsprofessoren Spyridon Labrou (1851-1919), Nikolaos Politis (1852-1921) und Georgios Chatzidakis (1848-1921) sowie der renommierte - deutschstämmige Jurist Konstantinos Hoesslin (1844-1920). Ein anderes wichtiges Mitglied war der griechische Botschafter bei der Hohen Pforte Nikolaos Mavrogordatos (1837-1903), der seinen europäischen Kollegen Informationen über die IMROGräueltaten in Makedonien zuspielte ${ }^{22}$ und die Verbindung zwischen den Gruppen in Athen und Konstantinopel herstellte. Andere Mitglieder wurden in verschiedenen europäischen Hauptstädten aktiv, indem sie für die Ziele der Organisation warben und Geld sammelten. Als inoffizielles Sprachrohr der »Gesellschaft « diente hauptsächlich die Athener konservative Tageszeitung

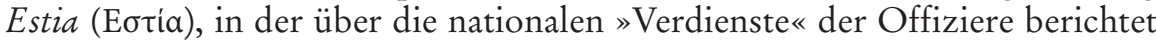
wurde. Ihr damaliger Herausgeber, der Dichter Georgios Drosinis (18591951), war selbst Mitglied. Bis 1897 zählte die Organisation circa 3.185 Mitglieder, die den unterschiedlichen Gesellschaftsschichten angehörten und aus verschiedenen Regionen Griechenlands und - nicht zu vergessen der griechischen Diaspora - kamen. Trotz der gesellschaftlichen Öffnung blieb ihr militärischer Charakter dennoch unangefochten, da sich die Offiziere weiterhin an den Ritualen und Strukturen der Armee orientierten ${ }^{23}$. Die Bewahrung der Anonymität ihrer Mitglieder verhalf der Organisation ferner dazu, ihr Ansehen bei der Bevölkerung zu erhöhen. Sie war tatsächlich eine »unsichtbare Kraft«, die überall und zu jeder Zeit »Präsenz« demonstrierte. Zugleich gewannen ihre Mitglieder den Eindruck, die »Auserwählten der Nation« zu sein, was ihr Selbstbewusstsein und die Bindungen untereinander stärkte.

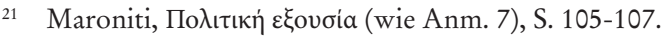

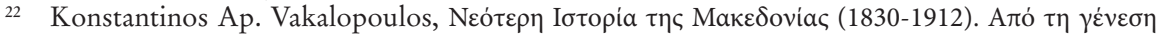

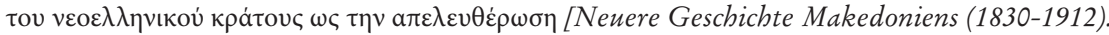
Von der Gründung des nengriechischen Staates bis zur Befreiung], 3. Aufl., Thessaloniki 2000, S. 247.

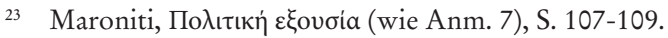


Die Gründung der »Nationalen Gesellschaft« war ein revolutionärer Akt auf halbem Wege: Zwar entstand sie aus der nationalistisch ausgerichteten Opposition gegen die Regierung und das Königshaus. Sie zielte aber weder auf die Abschaffung der bestehenden Institutionen noch auf die Errichtung einer Militärdiktatur oder die Etablierung einer neuen »patriotischen « Parteienlandschaft. Vielmehr wollte sie Thron, Parlament und Gesellschaft zum »nationalen Handeln « bewegen und auf ihren Kriegskurs einstimmen. Dennoch ersetzte sie bzw. beeinflusste sie inoffiziell die Regierung in außenpolitischen Fragen $^{24}$, woraus ein gefährliches Konfliktpotential erwuchs. Schwierig gestaltete sich auch ihr Loyalitätsverhältnis zum Königshaus, da der Kronprinz Konstantin I. (1868-1923) und seine Brüder führende Positionen im Generalstab innehatten ${ }^{25}$. Die Tatsache jedoch, dass die große Mehrheit der Offiziere die institutionelle Beteiligung des Königshauses an der Armeeführung ablehnte, verstärkte die oppositionelle Haltung der »Gesellschaft « gegenüber dem Thron. Daraus darf allerdings nicht geschlossen werden, dass alle Offiziere der Organisation antiroyalistisch eingestellt waren. Sogar diejenigen Offiziere, die für eine strikte Trennung von Armee und Königshaus plädierten, stellten die monarchische Staatsordnung nicht in Frage.

Für die »Nationale Gesellschaft « war die Nation »oberste Legitimationsinstanz $\ll^{26}$. In dieser Hinsicht verkörperte sie eine »politische Anomalie« im öffentlichen Leben, da ihre Offiziere den von ihnen auf die Gesetze des Landes geleisteten Eid nicht respektierten ${ }^{27}$. Ein ähnlicher Konfliktherd entstand innerhalb der Armee. Für die Subalternoffiziere der »Nationalen Gesellschaft « stellte ihre Mitgliedschaft einen weitaus höheren Wert dar, als ihr militärischer Rang innerhalb der Truppenhierarchie. Ihr Eid auf die Organisation und das Prinzip der Geheimhaltung setzten die militärischen Regeln de facto außer Kraft. Auf diese Weise wurde die Rangordnung der Streitkräfte unterhöhlt und eine Auseinandersetzung mit der Armeeführung riskiert. So bildete sich eine »Verschwörer«-Gruppe innerhalb der Armee, die eigene Regeln befolgte und Anordnungen von oben ignorierte ${ }^{28}$. Ihre Gründung demonstrierte zugleich die Unzufriedenheit einiger jüngerer Offiziere über verspätete oder ausgebliebene Beförderungen. Da die Offiziere der »Gesellschaft « enormen Einfluss innerhalb der Armee und der Politik ausübten, war es für ihre Vorgesetzten vermutlich nicht einfach, sie ihres Amtes zu entheben oder mit Sanktionen zu belegen. Außerdem sympathisierten viele von diesen mit den Zielen der Organisation. Das Zögern von Armeeführung und Regierung, gegen die Parallelstrukturen der »Gesellschaft « vorzugehen, vermittelte der Organisation das Gefühl, unbesiegbar zu sein und dazu bestimmt, das Schicksal der Nation in die eigene Hand zu nehmen. Der Staat tolerierte die

Zelepos, Die Ethnisierung (wie Anm. 19), S. 186.

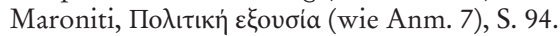

Zelepos, Die Ethnisierung (wie Anm. 19), S. 189.

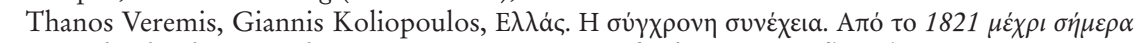
[Griechenland. Die moderne Kontinuität. Von 1821 bis heute], 2. Aufl., Athen 2006, S. 294-295.

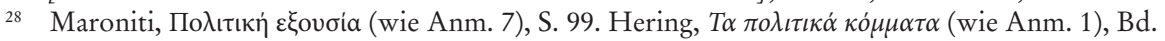
2, S. 708. 
Aktivitäten der »Gesellschaft « möglicherweise in der Hoffnung, sie bald kontrollieren zu können.

Unter der Leitung erfahrener »Kriegsfürsten«, das heißt ehemaliger Freischärler, die sich im Kampf gegen die osmanische Herrschaft ausgezeichnet hatten, bildete die »Gesellschaft« im Sommer 1896 irreguläre bewaffnete Einheiten, die nach Süd-Makedonien entsandt wurden und sich Gefechte mit den osmanischen Sicherheitskräften lieferten. Die Offiziere der »Gesellschaft« rekrutierten die »Banden «-Mitglieder und versorgten sie mit militärisch relevanten Informationen und Kriegsmaterial. Das hierfür benötigte Geld stammte aus Spenden der Mitglieder. Die Offiziere selbst waren am Guerillakampf nicht beteiligt, denn dies hätte wahrscheinlich ihren sofortigen Ausschluss aus der Armee bedeutet. Stattdessen begnügten sie sich mit der Vorbereitung des irregulären Kampfes. Das Ziel der "Banden« war es nicht, die griechisch fühlende Bevölkerung zum Aufstand gegen die Pforte anzustacheln, sondern der IMROTätigkeit entgegenzutreten und Konstantinopel von der Kreta-Krise abzulen$k^{2}{ }^{29}$. Die Anführer und Mitglieder dieser »Banden « waren hauptsächlich Flüchtlinge aus Makedonien und Epirus, die sich in Thessalien und in Athen niedergelassen hatten ${ }^{30}$. Sie bildeten die so genannte »Armee der Nation «, die eine Vorreiterrolle für die Befreiung der »unerlösten « Griechen spielen sollte ${ }^{31}$.

Die große Stunde der »Nationalen Gesellschaft« aber schlug ein halbes Jahr später, im Frühjahr 1897, als ein Aufstand der griechisch-orthodoxen Bevölkerung auf Kreta gegen die osmanische Herrschaft ausbrach. Vor dem Hintergrund der wieder aufflammenden Kreta-Frage, welche die Gemüter in Griechenland erregte, entsandte die »Nationale Gesellschaft« im Januar ein Memorandum an den König und die Regierung, in dem sie beide aufforderte, Maßnahmen zur Reorganisierung der Streitkräfte einzuleiten. Dabei handelte es sich um die offizielle Einmischung einer nichtstaatlichen Organisation in Regierungsangelegenheiten. Es war ein illegaler Akt und ein klarer Bruch mit der verfassungsmäßigen Ordnung ${ }^{32}$. Aus diesem Konflikt mit dem Staat ging die »Nationale Gesellschaft« als Sieger hervor, weil sie die Regierung Deligiannis' dazu zwang, auf Kriegskurs zu steuern. Dank der ihr ergebenen Presse war sie in der Lage, sich an die Bevölkerung zu wenden, patriotische Apelle zu veröffentlichen und jede oppositionelle Stimme als »verräterisch« zu brandmarken. Dem Druck der »Nationalen Gesellschaft«, der parlamentarischen Opposition und der Öffentlichkeit nachgebend, entschloss sich die Regierung Deligiannis' schließlich zu einer militärischen Intervention auf Kreta. Ohne diplomatische Unterstützung seitens der Großmächte ließ sie sich auf ein gefährliches Kriegsabenteuer ein, das mit einer demütigenden Niederlage Griechenlands endete ${ }^{33}$.

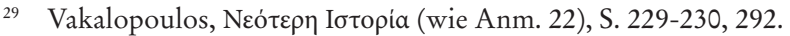

30 John Koliopoulos, Brigands with a Cause. Brigandage and Irredentism in Modern Greece 18211912, Oxford 1987, S. 215-216.

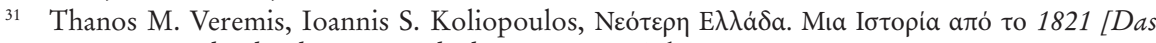
Nenere Griechenland. Eine Geschichte seit 1821], Athen 2013, S. 74-75.

32 Veremis-Koliopoulos, E $\lambda \lambda$ ás (wie Anm. 27), S. 294.

33 Vakalopulos, Griechische Geschichte (wie Anm. 1), S. 174-175. 
Zur selben Zeit beließ es die »Nationale Gesellschaft« nicht nur bei Appellen, sondern entsandte - vermutlich mit Zustimmung der Regierung - bewaffnete Formationen, die eine Art inoffizielle Vorhut der regulären griechischen Streitkräfte darstellten, nach Thessalien und Kreta. Anfang März 1897 übernahm die Gallionsfigur des späteren »Makedonischen Kampfes« Pavlos Melas die Organisierung der Freischärler-»Banden « in Thessalien mit Waffen, Munition und Klepbten-Kleidung ${ }^{34}$. Die Zahl dieser »Banden « wird auf circa dreißig geschätzt ${ }^{35}$. Zugleich wurden auf Betreiben der »Gesellschaft« Freiwillige und Waffen zu den Aufständischen auf Kreta geschmuggelt ${ }^{36}$. Für die Führung der Organisation - wie für die große Mehrheit der Bevölkerung - kam nur die Vereinigung der Insel mit dem griechischen Königreich als akzeptable Lösung in Frage. Die Gewährung eines Autonomie-Status für Kreta, wie es die Repräsentanten der Großmächte vorschlugen, wurde in Athen nicht ernsthaft in Erwägung gezogen.

Ende März 1897 überschritten die Freischärler-»Banden« der »Nationalen Gesellschaft « die griechisch-osmanische Grenze in Thessalien und lieferten sich Kämpfe mit regulären osmanischen Einheiten. Doch sehr bald wurden sie aufgerieben und nach Griechenland zurückgeworfen. Konstantinopel hatte nun einen wichtigen Grund, Athen den Krieg zu erklären ${ }^{37}$. Die osmanischen Truppen überrannten die griechischen Stellungen und besetzten die thessalische Ebene. Nur die politische Intervention der Großmächte, die um den Bestand der griechischen Monarchie fürchteten, verhinderte einen weiteren osmanischen Vormarsch. Im Friedensvertrag vom Dezember 1897 wurde Griechenland dazu verpflichtet, eine Kriegsentschädigung an die Pforte zu entrichten und eine Internationale Finanzkontrollkommission für die Tilgung seiner alten Schulden zu akzeptieren. Kreta erhielt die Autonomie, wenn auch unter osmanischer Oberhoheit.

Die »Schande von 1897 « hatte weitreichende Konsequenzen für die Organisation. Sie demoralisierte die Mitglieder der »Nationalen Gesellschaft « und führte schließlich zu ihrer Auflösung. Eine Welle der Entrüstung ging durch das ganze Land, und die Organisation wurde für den Kriegsausbruch und die darauf folgende Niederlage verantwortlich gemacht. Sogar diejenigen Zeitungen, welche zuvor die Ideen der »Nationalen Gesellschaft « verbreitet hatten, polemisierten nun gegen sie. In der Armee erhoben sich Stimmen gegen das »verantwortungslose Verhalten« der Organisation, die zum Sündenbock für die Niederlage wurde ${ }^{38}$. Die politische Opposition gegen Deligiannis, angeführt vom Populisten Dimitrios Rallis (1844-1921) ${ }^{39}$, verschwieg nunmehr ihre bisherige rhetorische Unterstützung für die »Gesellschaft « ${ }^{40}$. Auch einzelne Mitglieder gingen auf Distanz zur Organisation. Es war nicht mehr klug, sich mit ihr zu identifizieren.

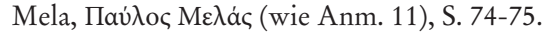

Koliopoulos, Brigands (wie Anm. 30), S. 220.

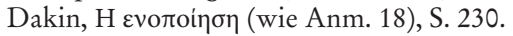

Zelepos, Die Ethnisierung (wie Anm. 19), S. 181.

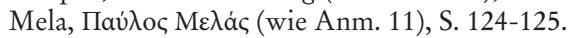

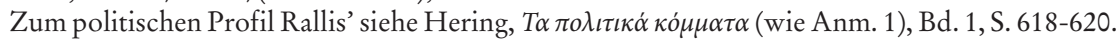

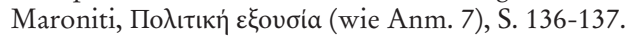


Wie reagierte die »Nationale Gesellschaft« auf die schweren Anschuldigungen? Am 22. April 1897 trat sie mit einem Aufruf an die Öffentlichkeit. Darin erklärte sie ihr Festhalten an dem von ihr vorgegebenen »nationalen « Kurs und beteuerte, dass sie nichts ohne Wissen der Regierung unternommen habe ${ }^{41}$ Letzteres entsprach sicher nicht der Wahrheit. Ihr Aufruf blieb jedoch ohne das erwünschte öffentliche Echo. Die Regierung zwang die Organisation, sich selbst aufzulösen. Daraufhin zogen sich viele Mitglieder ins Private zurück. Andere - beispielsweise Melas - hielten ihre Verbindungen zu anderen Mitgliedern aber aufrecht. Zwar gründeten sie keine neue Organisation; sie führten jedoch Gespräche miteinander über die Aufnahme des bewaffneten Kampfes in Makedonien gegen die IMRO, der 1904 stattfand $^{42}$.

Trotz ihres unrühmlichen Untergangs besaßen die Strukturen und Ziele der »Nationalen Gesellschaft« eine Vorbildfunktion, etwa für Organisationen des bürgerlich und konservativ geprägten Widerstands gegen die deutsche und bulgarische Besatzungsherrschaft in Makedonien (1941-1944)43. Keiner »Nachfolgeorganisation « aber gelang es auch nur annähernd, das Ansehen und die Bedeutung der »Nationalen Gesellschaft « zu erreichen. Für den Erfolg der »Gesellschaft « spielten mehrere Faktoren eine Rolle: Zunächst war sie eine aus bereits existierenden Militärstrukturen entstandene Geheimorganisation, die eine eigene Hierarchie und eigene Regeln sowie Rituale entwickelte. Das Prinzip der Geheimhaltung erwies sich als eine wichtige Voraussetzung für die Rekrutierung neuer Mitglieder. Sehr geschickt nutzte sie die bestehenden Verbindungen unter den Offizieren, um ihren Aktionsradius innerhalb der Armee zu vergrößern und dann öffentlich wirksam zu werden. Dazu brauchte sie den Einsatz ziviler Repräsentanten von Staat, Kultur und Gesellschaft, die ihr einen großen Dienst für die »Legitimierung « ihrer Ziele und Forderungen leisteten. Dass die »Nationale Gesellschaft « Thron, Parlament und Regierung unter immensen Druck setzte und auf die Außenpolitik Einfluss nehmen konnte, verdankte sie auch der herrschenden kriegsfreundlichen Stimmung in breiten Volksteilen, angeheizt durch Tageszeitungen, welche die Ansichten der »Nationalen Gesellschaft« propagierten. Hätte Griechenland den Krieg gegen das Osmanische Reich gewonnen und sich Kreta sowie makedonische Territorien einverleibt, so wäre die » Nationale Gesellschaft« als erfolgreichste konspirative Vereinigung seit der Gründung des neugriechischen Staates in die Geschichte eingegangen. Stattdessen wurde sie für die beschämende Kriegsniederlage verantwortlich gemacht und dämonisiert.

${ }_{41}$ Mela, Пaú̉oc Mèás (wie Anm. 11), S. 126.

42 Ebd., S. 173-174, 179.

43 Gemeint ist hier die ebenfalls von Armeeoffizieren gegründete »Panhellenische Befreiungsor-

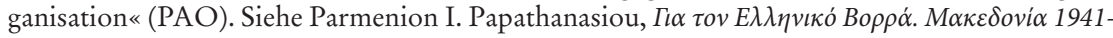

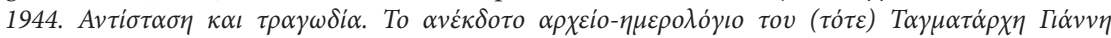
$\Pi \alpha \pi \alpha \theta \alpha v \alpha \sigma i o v$ [Für den griechischen Norden. Makedonien 1941-1944. Widerstand und Tragödie. Das unveröffentlichte Archiv-Tagebuch des (damaligen) Majors Giannis Papathanasiou], 2 Bde., 2. Aufl., Athen 1997. 